\title{
CORRESPONDENCE
}

\section{Taxonomy: add a human touch too}

Norman MacLeod and colleagues' call to develop automated species-identification systems is laudable (Nature $467,154-155 ; 2010$ ), but let's not forget a core feature of taxonomic work that depends on a scholar's input - the discovery of new characters.

Unexpected evolutionary novelties in morphology and physiology, for example, are what make taxonomic exploration rewarding. Taxonomists set out to discover and track such novelties and their evolutionary history. It is in this sense that taxonomy provides the empirical basis for understanding speciation and phylogeny.

There is a place for automated pattern detection, but it would not work with the 5,000 species of Drosophila, say, which are identified by their many different structures. Taxonomy can independently test and verify identifications without relying on patterns of single characters, as the long list of synonymies in any biological group testifies. The practice of taxonomic revision and publishing detailed monographs ensures that character distributions, species status and phylogenetic relationships are subject to repeated and critical testing.

We should beware the trend to confuse automatic identification tools with those that are useful for discovering new species. The emerging field of cybertaxonomy is an advance only if it is understood as enhancing and enabling theory-rich descriptive taxonomy, not replacing it.

As in many other modern scientific fields, including diagnostic medicine and molecular genetics, a final step involving a human expert is essential.

Antonio G. Valdecasas Museo
Nacional de Ciencias Naturales, CSIC, Spain,valdeca@gmail.com Quentin D. Wheeler Arizona State University, USA

\section{Taxonomy: include social networking}

Help with the shortage of professional taxonomists needed to identify organisms (Nature 467, 154-155;2010) may also come from an unexpected source social networking on the Internet.

Through social networking, the identification process can be made more efficient while simultaneously spreading real taxonomic knowledge. The facility is available to anyone, unlike other technologies that require specialized equipment.

In its first year of operation, the website iSpot (http://ispot. org.uk) has helped 6,000 users to identify 25,000 sightings of some 2,500 species, from lichens to birds. The website works by linking experts (including amateur experts) with beginners through a sophisticated reputation system that encourages users to help and learn from each other.

Eventually, DNA bar-code matching and image recognition might be added to the tools available. But these will be aids, not replacements, for people learning how to identify species. Jonathan Silvertown The Open University, UK, j.silvertown@open.ac.uk

\section{Call to reshape university curricula}

Universities worldwide are undergoing structural transformation, but reform of curricula is also key to their renewal. We invite scholars, educators and administrators to collaborate with us to reshape university teaching and learning.
Universities are experiencing a crisis of purpose, focus and content, rooted in a fundamental confusion about all three. The crisis is all the more visible as their pace of social, intellectual and technological change falls increasingly out of step with that outside. Furthermore, universities are largely reactive where they should be visionary and critical.

Curricula are mostly separated from research, and subjects are taught in disciplinary isolation. Knowledge is conflated with information and is too often presented as static rather than dynamic.

There can be no standard formula to rectify these problems, given the diversity of institutional structures and cultural differences among universities. However, our working group of scholars has made a start. To inform international dialogue and guide the experimental process of redesigning curricula, we have just published a set of 11 principles for rethinking undergraduate courses worldwide (see www.curriculumreform.org), and we welcome any suggestions.

Yehuda Elkana

Wissenschaftskolleg zu Berlin, Germany

Manfred D. Laubichler Arizona State University, USA, manfred.laubichler@asu.edu Adam S. Wilkins Centre de Recherches Interdisciplinaires, France

\section{Science as a threat to far-right fringe}

The scorn of the US far-right

'Tea Party' fringe for science, particularly relating to sustainability, climate change and biodiversity, stems from a perceived threat to its idealized views of how the world should be (Nature 467, 133; 2010).

The great (and undervalued) psychologist George Kelly described threat as "the awareness of an imminent comprehensive change in one's core structures". Science opens up our culture's core structures to criticism and calls their importance into question - one example being non-sustainable approaches to managing the planet.

The far-right's response to the threat posed by modern environmental science goes roughly like this. First, discredit a piece of scientific data the 'hockey-stick' graph, for example. When that fails to remove the threat of climatechange science, discredit the scientists (as happened in 'Climategate'). When that doesn't work either, discredit science as a method for understanding the world. Richard Kool Royal Roads University, British Columbia, Canada, rick.kool@royalroads.ca

\section{Alternative view of Serengeti road}

Andrew Dobson and colleagues overstate the argument against a road across the Serengeti National Park in Tanzania (Nature 467, 272-273; 2010). Migrant wildebeest already regularly cross transportation arteries in the Serengeti and in adjacent linked ecosystems. Established evidence of impacts on declines in migratory wildlife populations is centred not on roads, but on fencing - a separate and more remote possibility.

Conservation policy in the Serengeti has too often been based on idealized notions of what landscapes should look like. The Serengeti ecosystem has been shaped by thousands of years of human use, and is now groomed by park managers rather than by local inhabitants.

The ecosystem is supported and enhanced by pastoralist land use in surrounding buffer zones. 